IMMUNOTHERAPY

\section{Interferon in anti-CTLA-4 responses}

Immune-checkpoint blockade is a breakthrough in anticancer therapy, with dramatic sustained responses observed across a range of tumour types. Nevertheless, response rates are modest and acquired resistance can occur. IFN $\gamma$ is a hallmark cytokine of an antitumour immune response, and disruption of the IFN $\gamma$ pathway in tumour cells, particularly loss-of-function mutations in JAK1/JAK2, has been implicated in acquired resistance to programmed cell-death protein 1 blockade. A new study reveals that loss of IFN $\gamma$ signalling might also underlie intrinsic resistance to cytotoxic T-lymphocyte-associated protein 4 (CTLA-4) immune-checkpoint inhibition.

Corresponding author Padmanee Sharma explains the rationale for this study: "we previously conducted a pre-surgical study with anti-CTLA-4 antibodies in patients with bladder cancer, and found that treatment with these antibodies increased the abundance of an ICOS ${ }^{+}$T-cell subset that produced IFN $\gamma$. We hypothesized that tumour cells harbouring mutations in the IFN $\gamma$-signalling pathway would not be susceptible to the IFN $\gamma$ produced by these $T$ cells and, therefore, would be resistant to anti-CTLA-4 therapy."

The researchers tested this hypothesis by evaluating genomic alterations of IFN $\gamma$-pathway genes in 16 patients with melanoma, four 'responders' and 12 'non-responders' to treatment with the anti-CTLA-4 antibody ipilimumab. "We found that non-responders had tumour tissues harbouring loss of IFN $\gamma$-pathway genes at significantly higher frequencies than observed in responders," states

Sharma. Indeed, 184 alterations (an average of 15.33) were detected in non-responders, compared with only four (one on average) in responders $(P=0.015)$.

Of note, although no significant enrichment of single-nucleotide variants was observed, $75 \%$ of non-responders had copy-number alterations (CNAs) versus $0 \%$ of responders $(P=0.019)$. These findings were validated in an independent cohort, using previously published data. The CNAs were mostly gene losses, particularly of IFNGR1, IRF1, JAK2, and IFNGR2, but some amplifications involving inhibitors of IFN $\gamma$ signalling were also observed.

Using The Cancer Genome Atlas data, Sharma and co-workers found that patients with metastatic melanomas harbouring CNAs of IFN $\gamma$-pathway genes have shorter overall survival durations than those with tumours lacking such CNAs ( 40 months versus 48.2 months; $P=0.0018$ ). This finding indicates that disruption of IFN $\gamma$ signalling is associated with a poor prognosis, perhaps owing to reduced responsiveness of tumour cells to host antitumour immune responses.

"We plan to prospectively evaluate patients for responses to immune-checkpoint therapy based on the status of IFN $\gamma$-pathway genes in tumour tissues, and to determine whether combination therapy can effectively overcome the loss of IFN $\gamma$-pathway genes," Sharma concludes.

David Killock

ORIGINAL ARTICLE Gao, J. et al. Loss of IFN- $\gamma$ pathway genes in tumor cells as a mechanism of resistance to anti-CTLA-4 therapy. Cell http://dx.doi.org/10.1016/j.cell.2016.08.069 (2016) FURTHER READING Hutchinson, L. JAK - opening the door to acquired resistance. Nat. Rev. Clin. Oncol. 13, 528-529 (2016) 